\title{
Disseminated peritoneal leiomyomatosis - a rare complication of laparoscopic myomectomy with intraperitoneal morcellation
}

Grzegorz Kade', Sebastian Spaleniak², Emilia Frankowska³, Szczepan Cierniak, Agnieszka Gąsowska-Bodnar' ${ }^{1}$ Lubomir Bodnar ${ }^{5}$

'Warmian-Masurian Cancer Center of the Ministry of the Interior and Administration's Hospital in Olsztyn, Poland

${ }^{2}$ Department of Internal Medicine and Nephrodiabetology, Medical University of Lodz, Poland ${ }^{3}$ Department of Radiology, Military Institute of Medicine

${ }^{4}$ Department of Pathomorphology, Military Institute of Medicine

${ }^{5}$ Department Oncology at Faculty of Medical Sciences, University of Warmia and Masuria, Olsztyn, Poland

Correspondence:

Grzegorz Kade Warmian-Masurian Cancer Center of the Ministry of the Interior and Administration's Hospital in Olsztyn, Poland, phone: 895398000 e-mail: grzegorz.kade@poliklinika.net

Received: 24.07.2020 Accepted: 29.09.2020

DOI: 10.24292/01.OR.320290920 Copyright $\odot$ Medical Education. All rights reserved.

\section{ABSTRACT}

The present study reports the case of a 43-year-old female patient who presented with pulmonary benign metastasizing leiomyoma after a hysterectomy due to for leiomyoma of the uterus.

Key words: benign metastasising leiomyoma, myomectomy, myoma metastases 


\section{CLINICAL IMAGE}

A 43-year-old female patient was referred to an oncology clinic due to numerous metastatic lesions in/to the peritoneal cavity and simultaneous unclear disseminated nodular lesions in the lungs.

In the medical history, laparoscopic myomectomy with intraperitoneal morcellation due to symptomatic uterine myomas were diagnosed (2011). Afterwards, laparoscopic amputation of the body of the uterus without the adnexa due to myomas was performed (2013). Half a year later, due to persistent pain in the lower abdomen, an ultrasound of the abdominal cavity was per- formed, showing a well vascularized hyperechoic solid tumor. In order to assess the advancement of the disease, magnetic resonance (MR) with contrast of the chest, abdominal cavity and true pelvis was performed, revealing a heterogeneous nodular area (at the site of the removed uterus) (fig. 1A, B). Numerous synchronous nodular lesions in the lungs were simultaneusly described (2014) (fig. 1C, D). Imaging examinations of the chest performed before the surgery showed no pathology (2011). During the third operation, the cervix, bilateral adnexa, tumor (fig. 1E), mesenteric nodules and large intestine walls were removed. Bilateral lymphadenectomy was performed as well (2014).

\section{FIGURE 1A, B.}

Magnetic resonance imaging of the pelvis. In the hysterectomy site, a $66 \times 100 \mathrm{~mm}$ proliferative heterogeneous solid tumor, vascularized from the periphery, undergoing strong contrast enhancement. Free fluid in the recesses of the peritoneal cavity. T2-weighted images in axial (A) and sagittal (B) views.
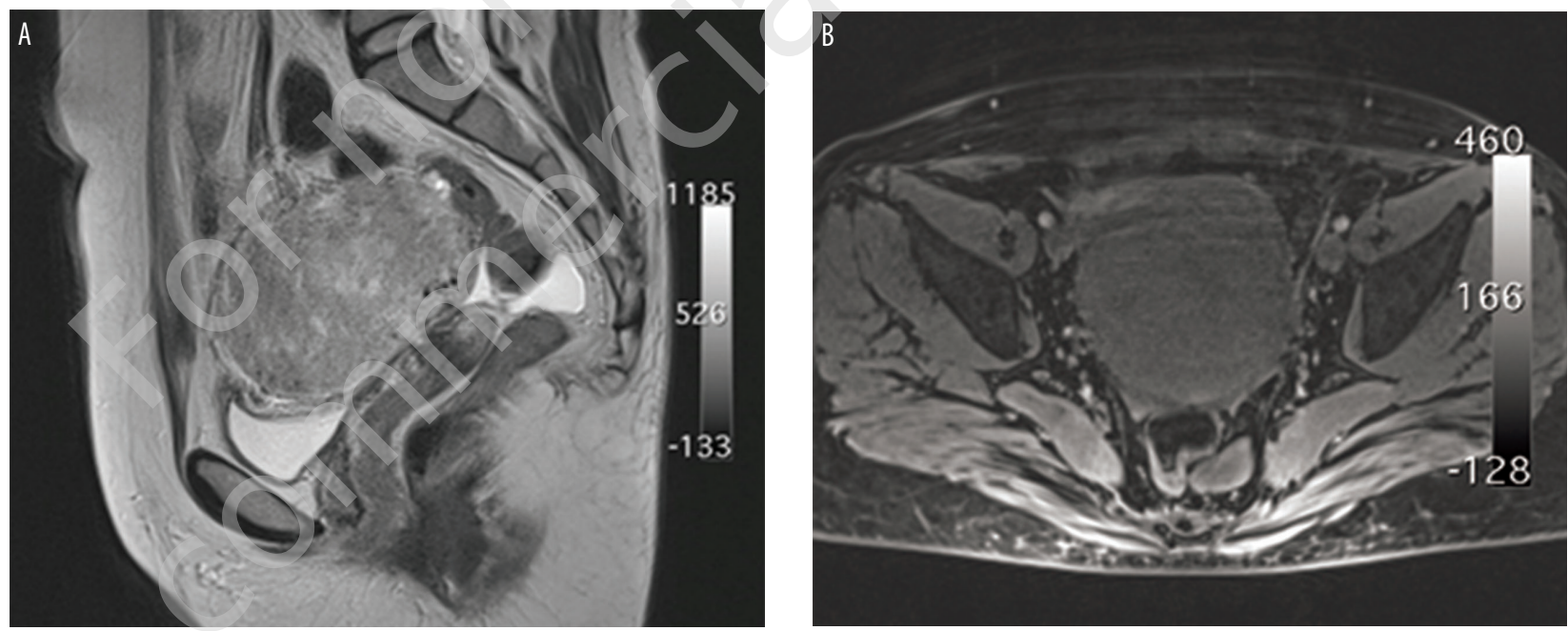

FIGURE 1C, D.

Magnetic resonance imaging of the chest (2014). Nodule in segment 6 of the left lung (arrow): hypointense in T2-weighted images, undergoing homogeneous enhancing after intravenous administration of a contrast agent. T2-weighted images in the axial view (A). T1-weighted images in the axial view after intravenous administration of a contrast agent (B).
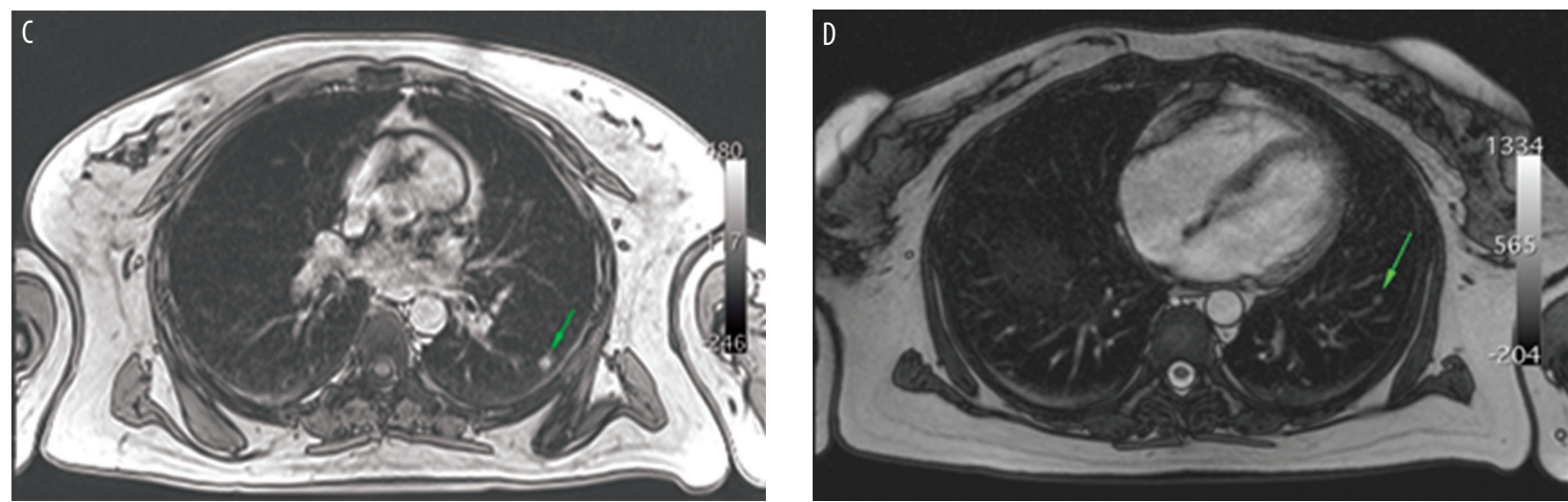
FIGURE 1E.

Magnetic resonance imaging of the abdominal cavity.

T2-weighted images in the axial view. Hypointense nodule associated with the parietal peritoneum (arrow).

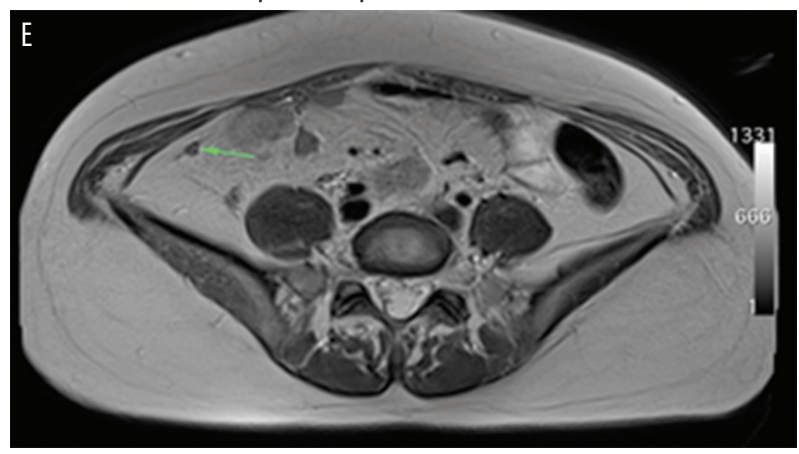

Based on the performed histopathological examinations, leiomyomatosis peritonealis disseminata (fig. 1F, G) was diagnosed. Due to the complex clinical picture and the uncertain nature of the lesions observed in the lungs, the pathomorphological reassessment of the specimens was performed.

FIGURE 1F.

Leiomyoma. $\mathrm{HE}$

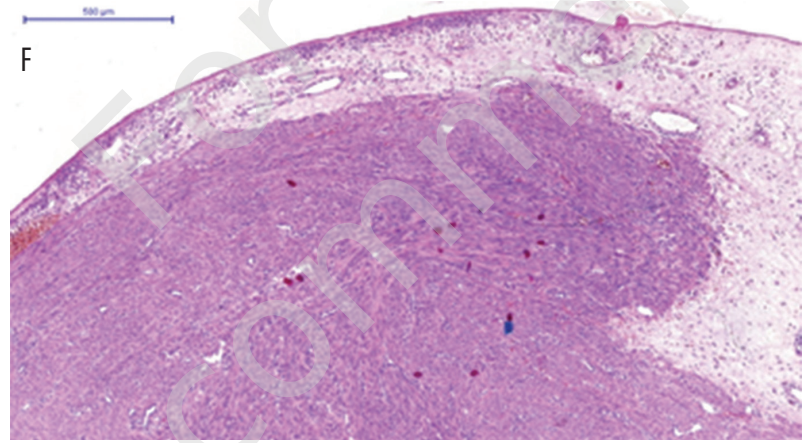

FIGURE 1G.

Peritoneal nodule with leiomyoma morphology. HE.

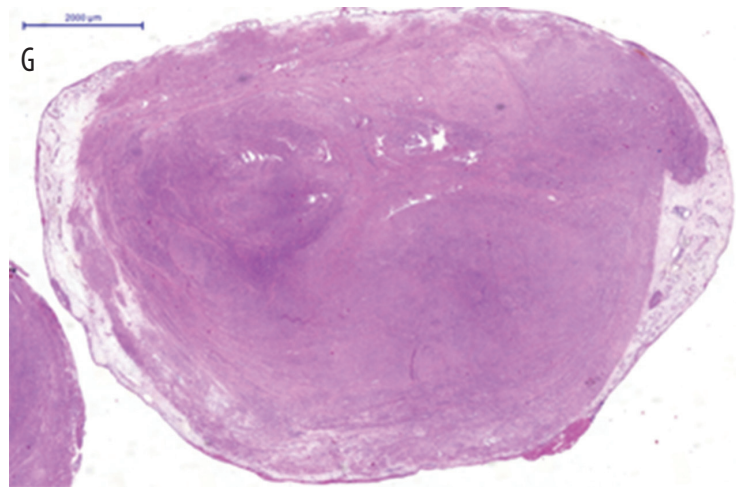

In the immunohistochemical study of peritoneal myoma tissue, EGFR(-), CD34(-) and CD11 (-) were found. The immunomorphological examination showed a positive reaction with smooth muscle antibodies and caldesmon (fig. 1H, 11). A low Ki-67 proliferation index of $<10 \%$ was observed (fig. 1J). After analyzing the specimens, the consulting pathologist confirmed the diagnosis of leiomyomatosis.

FIGURE $1 \mathrm{H}$.

Positive reaction to SMA (a-smooth muscle antibody).

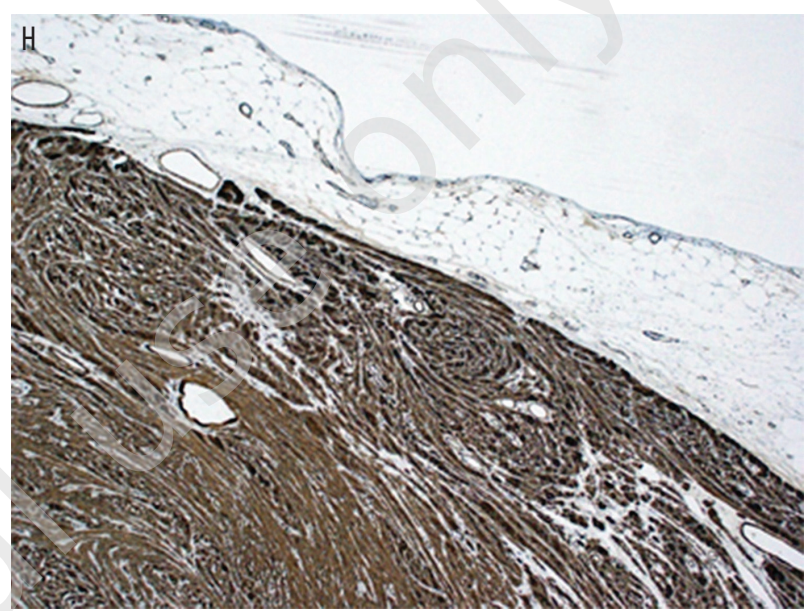

FIGURE 11.

Positive response to $\mathrm{h}$-caldesmon.

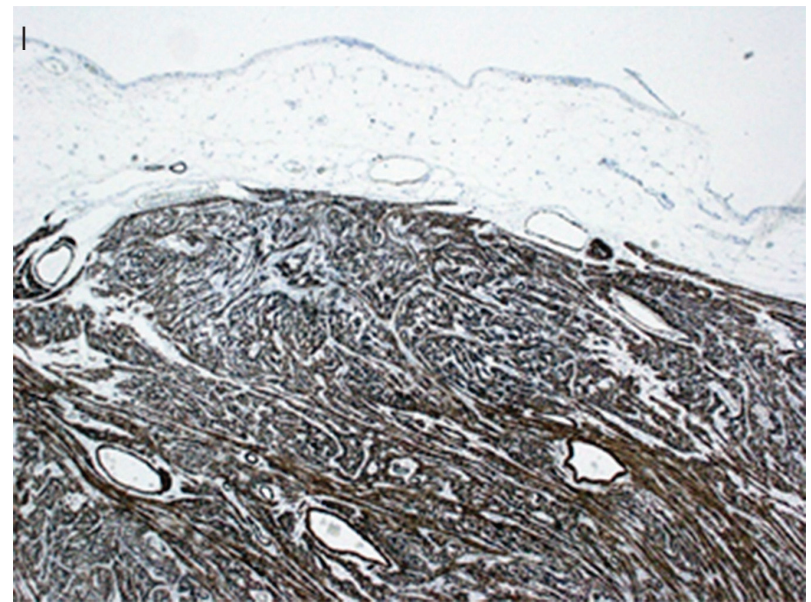

FIGURE $1 J$.

Ki-67 (below 3\%).

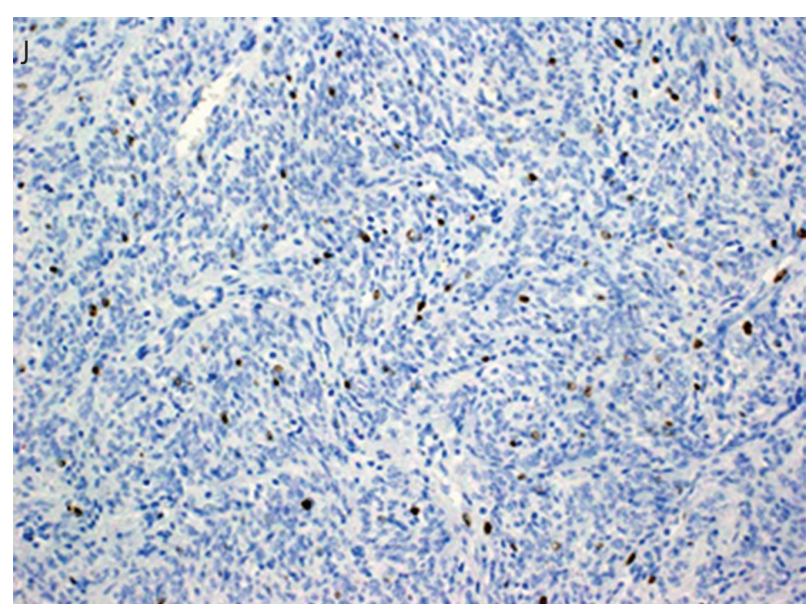


In order to evaluate nodular lesions in the lungs, a control chest computed tomography (CT) with contrast was performed. The PP described six 3 to $5 \mathrm{~mm}$ smooth-walled nodules of homogeneous density without calcification and features of decay, the PL seven similar nodules of size from 3 to $7 \mathrm{~mm}$ (2014), which might correspond to metastatic myomas.

Due to the location of the lesions, the lack of clinical symptoms, correct results of spirometry, body plethysmography and a stable image in the next CT of the chest (June 2015), invasive pneumological diagnosis was abandoned. In the control MR (June 2015) of the abdominal cavity, no signs of recurrence or renal angiomyolipoma (AML) were observed.

There are approximately 100 reported cases of benign metastasising leiomyoma (BML) in the literature [1]. The paper presents a very rare case of multiple myoma metastases to the peritoneal cavity and, most kilely, to the lungs. The described case is the first case of BML with concomitant peritoneal metastases and endometriosis.

Uterine myomas are among the most common benign tumors in women. In each case of nodular lesions, especially in the lungs of women after hysterectomy due to uterine myomas, the differential diagnosis should take into account the metastases of the myomas. A particularly high risk of metastasis is associated with the administration of the surgical method using intraperitoneal morcellation of myomas.

In response to reports that the risk of diagnosing an accidental leiomyoma/in patients undergoing surgery for myomas is approximately 1 in 352 cases, the US Food and Drug Administration (FDA) issued a warning in April 2014 against the use of intraperitoneal morcellation in the treatment of myomas [2].

The pathogenesis of myoma metastases to distant organs is still subject to various speculations. There are no guidelines due to the small number of cases. It has been shown that, apart from surgical and pharmacological castration, the use of tamoxifen (a selective estrogen-receptor modulator) and aromatase inhibitors reduces the tumor size [3].

Spontaneous regressions of pulmonary metastases have been reported in pregnant and postmenopausal women. This indicates that active treatment cannot be justified in asymptomatic patients [4]. In the case of suspected malignancy of a metastatic lesion, in addition to the biopsy of the lesion, PET/CT with 18-FDG seems to be a non-invasive differential test [5].

During the 5-year follow-up period, no recurrence of symptoms or progression of nodule size was found in subsequent $\mathrm{CT}$ and MR examinations.

\section{References}

1. Beck MM, Biswas B, D'Souza A et al. Benign metastasising leiomyoma after hysterectomy and bilateral salpingo-oophorectomy. Hong Kong Med J. 2012; 18: 153-5.

2. C. for D. and R. Health, Surgery Devices - Laparoscopic Power Morcellators (n.d.). https://www.fda.gov/medicaldevices/productsandmedicalprocedures/surgeryandlifesupport/ucm584463.htm (access: 25.02.2020).

3. Rivera JA, Christopoulos S, Small D et al. Hormonal manipulation of benign metastasizing leiomyomas: report of two cases and review of the literature. J Clin Endocrinol Metab. 2004; 89: 3183-8.

4. Wei WT, Chen PC. Benign metastasizing leiomyoma of the lung: a case report and literature review. Oncol Lett. 2015; 10(1): 307-12.

5. Sawai Y, Shimizu T, Yamanaka Y et al. Benign metastasizing leiomyoma and 18-FDG-PET/CT: A case report and literature review. Oncol Lett. 2017; 14(3): 3641-6.

Authors' contributions: Grzegorz Kade: 30\%; Sebastian Spaleniak: 30\%; Emilia Frankowska: 10\%; Szczepan Cierniak: 10\%; Agnieszka Gąsowska-Bodnar: 10\%; Lubomir Bodnar: 10\%. Conflict of interests: None.

Financial support: None. 